\title{
From buildings to point-line geometries and back again
}

go back

full screen

close

quit

\author{
Ernest Shult*
}

An hour talk given at the Conference on Buildings and Groups, held in Ghent, Belgium, May 20-26, 2007.

\begin{abstract}
A chamber system is a particular type of edge-labeled graph. We discuss when such chamber systems are or are not associated with a geometry, and when they are buildings. Buildings can give rise to point-line geometries under constraints imposed by how a line should behave with respect to the point-shadows of the other geometric objects (Pasini [P]). A recent theorem of Kasikova [K] shows that Pasini's choice is the right one. So, in a general way, one has a procedure for getting point-line geometries from buildings. In the other direction, we describe how a class of point-line geometries with elementary local axioms (certain parapolar spaces) successfully characterize many buildings and their homomorphic images. A recent result of $\mathrm{K}$. Thas [KT] makes this theory free of Tits' the classification of polar spaces of rank three [T1]. One notes that parapolar spaces alone will not cover all of the point-line geometries arising from buildings by the Pasini-Kasikova construction, so the door is wide open for further research with points and lines.
\end{abstract}

Keywords: buildings, chamber systems, point-line geometries, parapolar spaces, Lie incidence geometry

MSC 2000: 51E24

\footnotetext{
*The author is grateful to the organizers for their invitation and support, and even more grateful for the vital updated interchange this meeting provided for all participants.
} 


\section{Introduction}

This paper represents an attempt to place in perspective the relation between the theory of buildings and characterizations of point-line geometries bearing simple local axioms.

\section{Buildings}

Buildings are really chamber systems rather than geometries. Often there is a class of geometries that goes with a chamber system, and one may want to think of these geometries as the buildings; but really they are not the buildings. The latter are simply nice geometries — some met by geometers a century ago, some met by Greek geometers more than two thousand years ago- but they do not tell the real story. That role falls to chamber systems.

\subsection{Chamber systems}

A chamber system is a set of objects $C$, which we shall call "chambers", together with a mapping

$$
\lambda: \text { unordered pairs of distinct chambers } \rightarrow 2^{I},
$$

the set of all subsets of a set $I$ called the type set; the mapping $\lambda$ must satisfy this property: for any three-set of chambers $\{x, y, z\}$ one has

$$
\lambda(x, y) \cap \lambda(y, z) \subseteq \lambda(x, z)
$$

For any type $i \in I$, let us say that two distinct chambers $x$ and $y$ are $i$-adjacent if $i$ is a member of the set $\lambda(x, y)$. Then equation (1) implies that when combined with the identity relationship, $i$-adjacency becomes an equivalence relation which we denote by $i^{*}$. Any $i^{*}$-equivalence class is called an $i$-panel. ${ }^{1}$

Of course one may let $E$ be the collection of unordered pairs of chambers for which $\lambda$ assumes a non-empty value. Then we may regard $C=(C, E)$ as a simple graph for which each edge $e$ is assigned a non-empty set of types $\lambda(e)$ such that equation (1) holds. We say that the chamber system $(C, E, \lambda)$ is connected if and only if the graph $(C, E)$ is connected.

The collection of all chamber systems over the set $I$ forms a category when provided with morphisms $f$ which are graph morphisms such that the typeset

\footnotetext{
${ }^{1}$ This definition is equivalent to the one given in Tits' book as a system $\left\{\pi_{i}\right\}$ of (not-necessarily distinct) partitions of $C$ indexed by elements of $I$.
} 




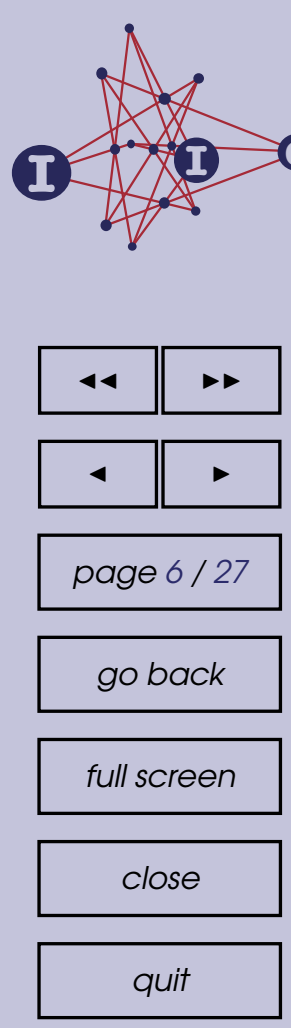

Theorem 2.2 (Arjeh Cohen $[\mathrm{BrC}]^{6}$ ). (1) If a geometry $G$ is residually connected of finite rank, then so is the chamber system $\mathbf{C}(G)$, and there is a geometry isomorphism $\mathbf{\Gamma}(\mathbf{C}(G)) \simeq G$.

(2) If $C$ is a residually connected chamber system, then $\Gamma(C)$ is residually connected, and $\mathbf{C}(\boldsymbol{\Gamma}(C)) \simeq C$.

(3) There exists an isomorphism between the subcategory of residually connected geometries over a finite typeset $I$, and the subcategory of residually connected chamber systems over the same finite $I$.

Upon first reading, it would seem that there is a slight asymmetry between the first two statements of the Theorem. Assertion (1) entails finite rank in its hypothesis while Assertion (2) does not. Does the second assertion really apply in the more general realm of chamber systems of infinite rank? The answer is no. Consider:

Theorem 2.3 (Kasikova and Shult [KS1]). If $C$ is a chamber system over an infinite set $I$ each of whose panels contain at least two chambers, then $C$ is not residually connected. In particular, no building of infinite rank (definitions of these terms will appear below) is residually connected.

Put another way, if $C$ is a residually connected chamber system with all panels having at least two chambers, then it has finite rank, thus restoring symmetry to the first two statements of Theorem 2.2.

But there is a larger meaning to be read from Theorem 2.3, for it reveals a basic rupture between geometries and chamber systems once one ventures into infinite rank. In fact the two categories seem to live separate lives at infinite rank. On the one side, there are buildings (defined as chamber systems) at any conceivable rank; and on the other side, there are also classical geometries (such as projective spaces, polar spaces and certain Grassmannians of infinite singular rank) which exist and can be characterized, but cannot find a chamber system building to latch onto.

\subsection{Buildings as Chamber Systems}

\subsubsection{Chamber systems of type $M$}

Let $M$ be a symmetric matrix whose whose rows and columns are indexed by $I$, and whose entries are positive integers or the symbol " $\infty$ ". It is required that the

\footnotetext{
${ }^{6} \mathrm{~A}$ necessary and sufficient condition that a chamber system have the form $\mathbf{C}(\Gamma)$, is given in Proposition 12.34 of [P]. It does not necessarily imply the isomorphism of the second statement of this Theorem.
} 





\subsection{More general constructions}

Once again, we assume that we have a building $B$ which, in the finite rank case, will be regarded as both a geometry over $I$ as well as a chamber system over $I .{ }^{11}$ Our objective is to select a subset $J$ of the typeset, and think of the flags of type $J$ in the building geometry as the set of "points" of a geometry. There are two issues: (i) what are the other objects that we should be considering? (ii) How do we make a reasonable point-line geometry with the flags of type $J$ as points?

full screen

close quit

ACADEMIA PRESS

N

$\widehat{\underline{\text { IIIIII }}}$ UNIVERSITEIT GENT

\subsubsection{The geometry of $J$-reduced objects}

If $F$ is any flag of the building geometry $B$, the $J$-shadow of $F$ is simply the collection $\operatorname{sh}_{J}(F)$ of all flags of type $J$ which are incident with the flag $F$. (Recall that in a geometry, two flags are defined to be incident if and only if their union is also a flag.) The problem is that sometimes there are geometric objects which are members of a flag which are not essential in determining the shadow of that flag. Thus we could say that an object $x \in F$ is inessential relative to $F$ if the $J$-shadow of $F-\{x\}$ is the same as that of $F$; and that $x$ is essential relative to $X$ otherwise. The point is that if $Y$ is any sub-flag of $X$ which contains $x$ and if $Y$ "still supports the $J$-shadow of $X$ " - that is, $\operatorname{sh}_{J}(F)=\operatorname{sh}_{J}(Y)$ - then, $x$ is essential to $Y$ as well. It follows that there is a a set $X_{J}$ of elements of $X$ which are essential to every subset of $X$ which supports the shadow of $X$ and moreover that any such supporting set $Y$ must contain all of these essential elements. Thus for every flag $F$ in the geometry $B$, there exists a subflag $r_{J}(F)$ consisting of only the $J$-essential objects. Such a flag is said to be $J$-reduced.

The next observation if that this reduction can be done universally in the poset of types. Thus, for any flag $F$ of type $K$, the $J$-reduced object $r_{J}(F)$, always has the same type $r_{J}(K)$. Thus the idempotent operator on the poset of flags that takes each flag to its $J$-reduced subflag is actually induced by a similar idempotent $\rho_{J}$ on the homomorphic image of the flag-poset under the typ homomorphism. Thus we have

$$
\operatorname{typ} \circ r_{J}=\rho_{J} \circ \text { typ }
$$

as poset morphisms from the poset of flags of the building geometry $B$ to the Boolean poset set of all subsets of $I$.

All of this is contained in Chapter 12 of Tits' book [T1], an appendix entitled "Shadows". There, a numbered complex plays the role of the flag complex

\footnotetext{
${ }^{11}$ In the infinite rank case, we must think of $B$ as a chamber system. We need both points of view in order to render historical presentations in their original language.
} 




\section{From point-line geometries to buildings}

\subsection{Introduction}

Now we consider the opposite endeavor: beginning with a point-line geometry subject to certain simple axioms on points and lines, can we recognize it as a truncation of some well known geometry? Throughout we shall assume each line possesses at least three points. ${ }^{14}$

\subsection{Two classic cases}

Theorem 4.1 (Projective spaces - Veblen-Young [VY]). If $(\mathcal{P}, \mathcal{L})$ is a linear space with at least two (thick) lines, and if $(\mathcal{P}, \mathcal{L})$ satisfies the Veblen axiom ${ }^{15}$, then it is either a projective plane or it is isomorphic to the geometry of 1- and 2-dimensional spaces of a (possibly infinite-dimensional) right vector space $V$.

Theorem 4.2 (Polar spaces - Veldkamp [V1], Tits [T1], Buekenhout-Shult [BS], Johnson [J], Johson-Pasini-Cuypers [CJP]). In $\Gamma=(\mathcal{P}, \mathcal{L})$ suppose only

(i) no point is collinear with all other points, and

(ii) for any non-incident point-line pair $(p, L), p$ is either collinear with exactly one point of $L$ or is collinear with all the points of $L$.

Then $\Gamma$ is one of the following:

(1) a generalized quadrangle (rank 2 polar space),

(2) a rank three polar space (classified by J. Tits in [T1]), or

(3) the geometry of 1- and 2-dimensional subspaces of a right vector space $V$ which are either all such isotropic spaces with respect to a non-degenerate reflexive sesquilinear form $f$ on $V$, or are all such subspaces which are totally singular with respect to a non-degenerate pseudoquadratic form $q$ on $V$.

[As the language of Theorem 4.2 suggests, a point-line geometry satisfying the hypotheses (i) and (ii) is called here a polar space (actually a non-degenerate polar space) in general contexts). It is not assumed in advance to be a partial linear space. Nor is it assumed that the singular subspaces are projective. Both of these statements can be proved using a theory of Teirlinck [Te]. The (polar)

\footnotetext{
${ }^{14}$ Some of the characterization theorems have versions which allow lines with two points, but we omit them in order to keep things simple.

${ }^{15}$ Sometimes called Pasch's axiom.
} 


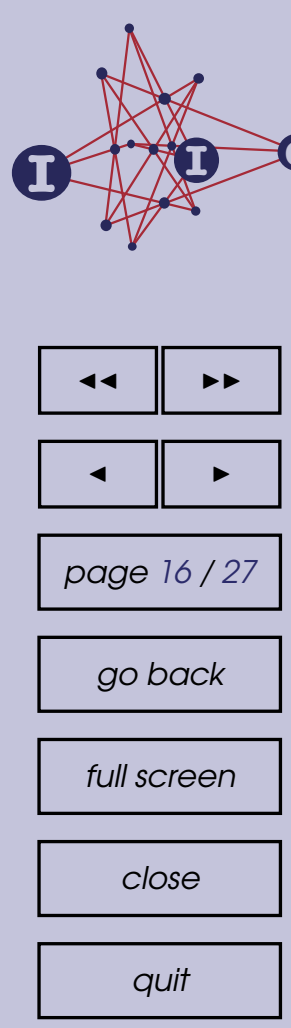

rank of a polar space is the rank of its geometry of singular subspaces when that number is finite, or is simply said to "infinite" otherwise.]

In theorems 4.1 and 4.2, the rank two cases (representing generalized 3-gons and 4-gons, respectively) have not been classified. ${ }^{16}$ The classification of the rank three polar spaces exploits the Moufang property, and parameterizes the spaces by norms on Cayley-Dickson algebras. I do not think it is an easy proof.

When the rank is beyond 2, both cases give us big groups - even when the enriched geometry of subspaces has infinite rank. For projective spaces, this is ensured by the Jacobson density theorem; for rank three polar spaces one has the Moufang property, and for classical polar spaces this is ensured by the infinite version of Witt's theorem which tell us that isometries between finite dimensional subspaces always lift to an isometry of $(V, f)$ or $(V, q)$ as appropriate. Please note that isometries between infinite-dimensional subspaces of $V$ need not lift. There are easy examples of sesquilinear forms $(V, f)$ which possess maximal singular subspaces of two different infinite dimensions, and one cannot lift an isometry of the smaller into the larger.

In both theorems, the finite rank examples are residually connected and their associated chamber systems are indeed buildings belonging to diagrams $A_{n}, B_{n}, C_{n}, D_{n}$. But what happens when there are singular subspaces of infinite projective rank? Are they buildings?

In answering this question one has to ask what are the objects in the geometry? For the sake of discussion, consider a sesquilinear form $(V, f)$ which has isotropic subspaces of infinite dimension. If one considers all isotropic subspaces to be objects of the geometry, we have a problem constructing the desired chamber system. True, unrefinable chains of subspaces exist (by a Zornification on the poset of chains ) but how does one define $i$-adjacency when there is an ambiguity about assigning types by dimension? On the other hand, if one solves the problem of types by considering only isotropic subspaces of finite dimension to be the objects of the geometry, how does one prove residual connectedness, a definition that refers to flags of corank one? It is enough to give you a headache.

\subsection{How point-line characterizations take place}

There are many interesting point-line geometries. For some - such as generalized quadrangles - it is impossible to increase the rank of the geometry by adding new classes of subspaces. In these cases one hopes that postulating groups of automorphisms may help. Most spectacular in this direction is the

\footnotetext{
${ }^{16}$ A classification is not at all likely in the case of planes, but the tightness of the situation seems to increase for quadrangles.
} 





\section{$1 \frac{1}{1}$
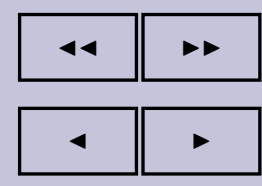 \\ (3) A polar Grassmannian of singular $\mathrm{PG}(k)^{\prime} s, k>1$, in a non-degenerate polar space of finite polar rank at least $k+2>4$.}

page 22 / 27

go back

full screen

close

quit

This theorem has its origin in a paper of Cohen [C1] characterizing metasymplectic spaces, which first introduced the pentagon property. (Of course, when speaking of "origins", all the theorems just listed must be played against the background of Tits' characterizations of these geometries (sometimes as point-line geometries rather than buildings, as in the case of polar spaces and metasymplectic spaces). The polar Grassmannian conclusion requires the use of "Hanssens' principle" [S6, Chapter 13] and Tits' "local approach theorem" [T2].

\subsection{Characterizations by singular subspaces}

Of course we have not covered all the spherical Lie-incidence geometries whose points are the objects whose type is represented by an end-node of the spherical diagram. We are missing $E_{7,2}, E_{8,1}$ and $E_{8,2}$. These and many non-spherical geometries of type $M$, whose points are represented by a single node, can be characterized as parapolar spaces with special conditions regarding the relation of points and a class of maximal singular subspaces (not necessarily all maximal singular subspaces).

One begins with a class $\mathcal{M}$ of maximal singular spaces of a parapolar space of symplectic rank at least 3 , and one supposes that there exists a positive integer $d$ such that for every pair $(x, M) \in \mathcal{P} \times \mathcal{M}, x^{\perp} \cap M$ is either empty or a PG $(d)$. Then $d=1$ or 2 . In the case that $d=1$, one must assume that there exists a line incident with at least two members of $M$. The conclusions are polar spaces, Grassmannians, Grassmannians mod a polarity, and half-spin geometries. Next, taking such a space to represent the point-residuals of a parapolar space of symplectic rank at least 4, one can show that there is a uniform outcome for residuals, thus yielding a polar space, or a geometry which is locally a Grassmannian, or a twisted Grassmannian modulo a polarity, or locally a homomorphic image of a half-spin geometry. All of these cases yield geometries that are homomorphic images of building geometries or a building geometry modulo a diagram polarity. (In the latter case, one must use Tits' local approach theorem on certain covers that admit the diagram polarity.) In this way, we obtain:

Theorem 4.7 ([S6, Chapter 16], [S4, S5]). Suppose $\Gamma=(\mathcal{P}, \mathcal{L})$ is a parapolar space of symplectic rank at least four having a class of maximal singular subspaces $\mathcal{M}$ such that every plane is contained in a member of $\mathcal{M}$, and for which there exists 

[BSch] F. Buekenhout and W. Schwarz, A simplified version of strong connectivity in geometries, J. Combin. Theory Ser. A 37 (1984), 73-75.

[BS] F. Buekenhout and E. Shult, On the foundations of polar geometry, Geom. Dedicata 3 (1974), 155-170.

[C1] A. Cohen, An axiom system for metasymplectic spaces, Geom. Dedicata 12 (1982), 417-433.

[C2] , On a theorem of Cooperstein, European J. Combin. 4 (1983), 107-106.

[CC] A. Cohen and B. Cooperstein, A characterization of some geometries of Lie type, Geom. Dedicata 15 (1983), 73-105.

[CS] A. Cohen and E. Shult, Affine polar spaces, Geom. Dedicata 35 (1990), 43-76.

[Cp] B. Cooperstein, A characterization of some Lie incidence structures, Geom. Dedicata 6 (1977), 205-258.

[CP] B. Cooperstein and A. Pasini, The non-existence of ovoids in the dual polar space $D W(5, q)$, J. Combin. Theory Ser. A 104 (2003), 351-364.

[Cu1] H. Cuypers, On a generalization of Fischer spaces, Geom. Dedicata 34 (1990), 67-87.

[Cu2] _ Affine Grassmannians, J. Combin. Theory Ser. A 70 (1995), 289304.

[CJP] H. Cuypers, P. Johnson and A. Pasini, On the classification of polar spaces, J. Geom. 48 (1993), 56-62.

[CP] H. Cuypers and A. Pasini, Locally polar geometries with affine planes, European J. Combin. 13 (1992), 39-57.

[DB] B. De Bruyn, Generalized quadrangles with a spread of symmetry, European J. Combin. 20 (1999), 759-771.

[DS] A. W. M. Dress and R. Scharlau, Gated sets in metric spaces, Aequationes Math. 34 (1987), 112-120.

[ES] C. A. Ellard and E. Shult, A characterization of polar Grassmann spaces, preprint, Kansas State University, 1988.

[HW] J. Hemmeter and A. Woldar, Classification of the maximal cliques of size $\geq q+4$ in the quadratic forms graph in odd characteristic, European J. Combin 11 (1990), 433-449. 
[J] P. Johnson, Polar spaces of arbitrary rank, Geom. Dedicata 35 (1990), 229-250.

[K] A. Kasikova, Characterizations of some subgraphs of the pointcollinearity graphs of building geometries, European J. Combin. 28 (2007), 1493-1529.

[KS1] A. Kasikova and E. Shult, Chamber systems which are not geometric, Comm. Algebra 24 (1996), 3471-3481.

[KS2] _ Point-line characterizations of Lie geometries, Adv. Geom. 2 (2002), 147-188.

[P] A. Pasini, Diagram Geometries, Oxford Science Publications, Clarendon Press, Oxford, 1994.

[Sch] R. Scharlau, A characterization of Tits buildings by metrical properties, J. London Math. Soc. 32 (1985), 317-327.

[S1] E. Shult, Characterizations of the Lie incidence geometries, in Surveys in Combinatorics, London Math. Soc. Lecture Note Ser. vol. 82, ed. E. Keith Lloyd, pp. 157-186, Cambridge University Press, Cambridge, 1983.

[S3] Characterizations of spaces related to metasymplectic spaces, Geom. Dedicata 30 (1991), 325-371.

[S2] - Aspects of buildings, in Groups and Geometries (Siena, 1996), Lino Martino et al (eds.), pp. 177-188, Birkhäuser, Basel, 1998.

[S4] Characterizing the half-spin geometries by a class of singular subspaces, Bull. Belg. Math. Soc. Simon Stevin 12 (2005), 883-894.

[S5] Characterization of Grassmannians by one class of singular subspaces, Adv. Geom. 3 (2003), 227-250.

[S6] , Points and Lines: Characterization of the Lie Incidence Geometries, book submitted for publication.

[KT] E. E. Shult and K. Thas, A theorem of Cohen on parapolar spaces, Combinatorica, to appear.

[Te] L. Teirlinck, Planes and hyperplanes of 2-coverings, Bull. Belg. Math. Soc. Simon Stevin 29 (1977), 73-81.

[TPM] J. A. Thas, S. E. Payne and H. Van Maldeghem, Half Moufang implies Moufang for finite generalized quadrangles, Invent. Math. 105 (1991), 153-156. 


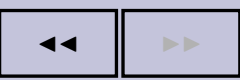

[T1] J. Tits, Buildings of Spherical Type and Finite BN-Pairs, Lecture Notes in Math., vol. 386, Springer, Berlin, 1974.

[T2] A local approach to buildings, in The Geometric Vein (the Coxeter Festschrift), C. Davis and B. Grunbaum, and F. A. Sherk (eds.), pp. 519547, Springer, Berlin, 2002.

[TW] J. Tits and R. M. Weiss, Moufang Polygons, Springer Monogr. Math., Springer, Berlin, 2002.

full screen

[VY] O. Veblen and J. Young, Projective Geometry, Ginn, Boston, 1916.

[Vl] F. D. Veldkamp, Polar geometry, I - IV, Indag. Math. 21 (1959), 512-551.

Ernest Shult

DePt. of Math., Kansas State UniV., Manhattan KS, 66502, USA

e-mail: ernest_shult@yahoo.com 